\section{Newly developed dual topoisomerase inhibitor P8-D6 is highly active in ovarian cancer}

\author{
Inken Flörkemeier, Tamara N. Steinhauer, Nina Hedemann, Magnus Ölander, \\ Per Artursson, Bernd Clement and Dirk O. Bauerschlag
}

\begin{abstract}
Background: Ovarian cancer (OvCa) constitutes a rare and highly aggressive malignancy and is one of the most lethal of all gynaecologic neoplasms. Due to chemotherapy resistance and treatment limitations because of side effects, OvCa is still not sufficiently treatable. Hence, new drugs for OvCa therapy such as P8-D6 with promising antitumour properties have a high clinical need. The benzo[c]phenanthridine P8-D6 is an effective inductor of apoptosis by acting as a dual topoisomerase I/II inhibitor.

Methods: In the present study, the effectiveness of P8-D6 on OvCa was investigated in vitro. In various OvCa cell lines and ex vivo primary cells, the apoptosis induction compared with standard therapeutic agents was determined in two-dimensional monolayers. Expanded by three-dimensional and co-culture, the P8-D6 treated cells were examined for changes in cytotoxicity, apoptosis rate and membrane integrity via scanning electron microscopy (SEM). Likewise, the effects of P8-D6 on non-cancer human ovarian surface epithelial cells and primary human hepatocytes were determined.

Results: This study shows a significant P8-D6-induced increase in apoptosis and cytotoxicity in OvCa cells which surpasses the efficacy of well-established drugs like cisplatin or the topoisomerase inhibitors etoposide and topotecan. Non-cancer cells were affected only slightly by P8-D6. Moreover, no hepatotoxic effect in in vitro studies was detected.

Conclusion: P8-D6 is a strong and rapid inductor of apoptosis and might be a novel treatment option for OvCa therapy.
\end{abstract}

Keywords: apoptosis, chemotherapy, drug development, dual topoisomerase inhibitor, hepatotoxicity, OvCa

Received: 12 August 2021; revised manuscript accepted: 27 October 2021.

\section{Background}

Ovarian cancer (OvCa) is the fifth leading cause of cancer deaths among women and the most lethal gynaecological malignancy in the developed world. ${ }^{1}$ The frequent diagnosis in advanced stages and insufficient treatment options due to chemotherapy resistance and side effects lead to a poor prognosis. First line therapy usually consists of surgical cytoreduction followed by platinum/taxane-based combination chemotherapy, but most patients relapse with drug-resistance in the course of the disease. ${ }^{2}$ Previous studies demonstrated a benefit in second line therapy with topoisomerase inhibitors like liposomal doxorubicin or topotecan. However, response duration was commonly short. ${ }^{3}$ Consequently, an important aim is to reduce mortality by improved new therapeutic options.

P8-D6 acts as a dual topoisomerase poison by stabilizing the covalent Topo-DNA-intermediate of both topoisomerase (Topo) enzymes I and II. ${ }^{4}$ Topoisomerases regulate torsional stress in DNA to enable essential genome functions (e.g. transcription, replication or recombination). Topo I
Ther Adv Med Oncol

2021, Vol. 13: 1-11

DOI: 10.1177/

17588359211059896

(c) The Author(s), 2021. Article reuse guidelines: sagepub.com/journalspermissions
Correspondence to: Dirk 0. Bauerschlag Department of

Gynaecology and

Obstetrics, University Medical Centre Schleswig-

Holstein, Campus Kiel,

Arnold-Heller-Str. 3,

24105 Kiel, Germany

dirk.bauerschlag@uuksh.

de

Inken Flörkemeier

Department of

Gynaecology and

Obstetrics, University

Medical Centre Schleswig-

Holstein, Kiel, Germany

Department of

Pharmaceutical and

Medicinal Chemistry,

Christian-Albrechts-

University Kiel,

Pharmaceutical Institute,

Kiel, Germany

Tamara N. Steinhauer

Bernd Clement

Department of

Pharmaceutical and

Medicinal Chemistry,

Christian-Albrechts-

University Kiel,

Pharmaceutical Institute,

Kiel, Germany

Nina Hedemann

Department of

Gynaecology and

Obstetrics, University

Medical Centre Schleswig-

Holstein, Kiel, Germany

Magnus Ölander

Per Artursson

Department of Pharmacy,

Uppsala University,

Uppsala, Sweden 


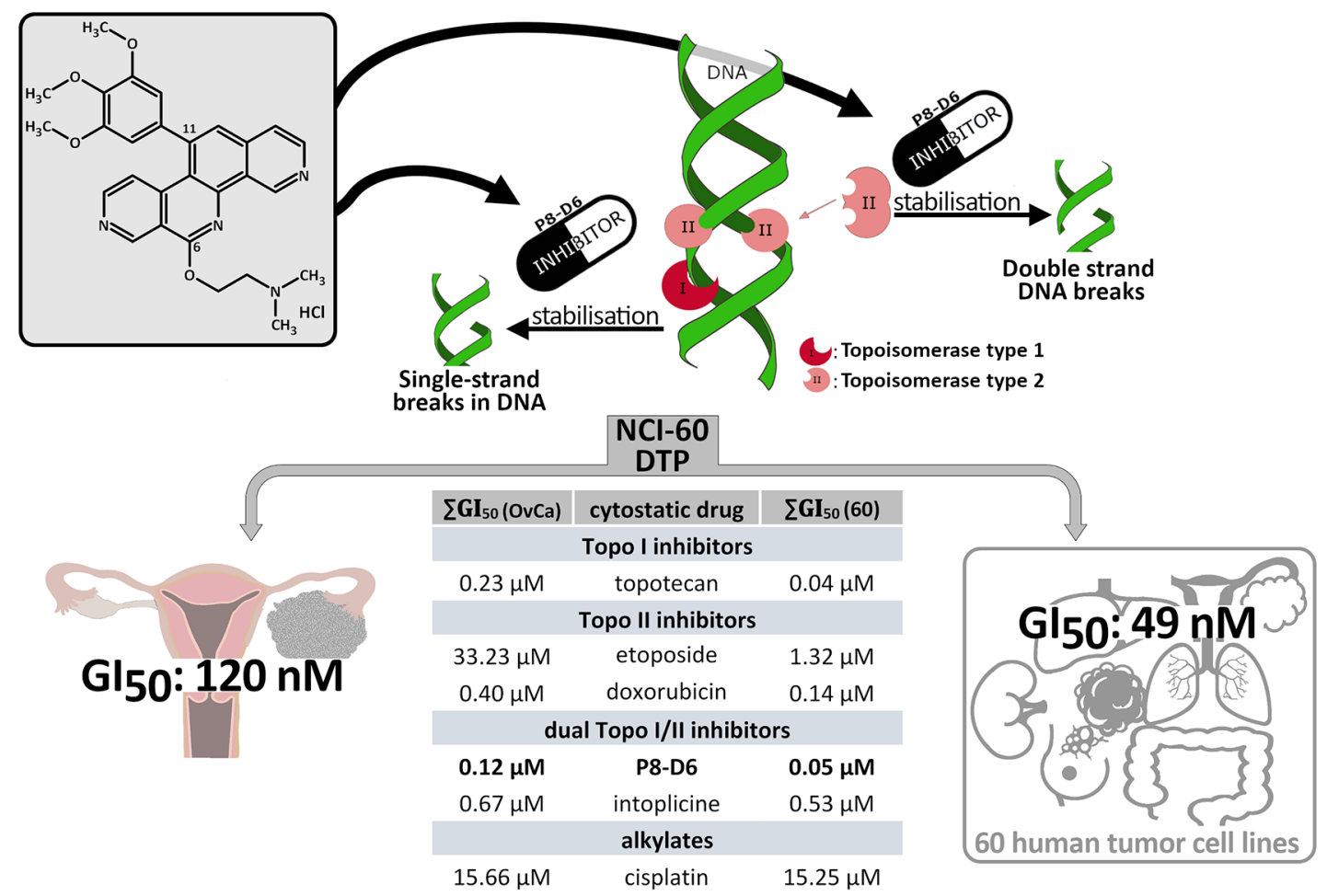

Figure 1. Chemical structure and mechanism of P8-D6 action. P8-D6 acts as a dual topoisomerase inhibitor by stabilizing the cleavable Topo-DNA complex, thereby inducing apoptosis. The effectiveness and the broad activity spectrum of P8-D6 were examined for the first time in a 60 -tumour cell line panel by the $\mathrm{NCl}$. In the evaluation, P8-D6 reached an average $\Sigma G_{50}(60)$ value of $49 \mathrm{nM}$ in multiple tumour cell lines. ${ }^{7}$ For the ovarian carcinoma cells tested, this $\mathrm{GI}_{50}$ average was $0.12 \mu \mathrm{M}$. For comparison, other active drugs are listed. $\Sigma \mathrm{GI}_{50}(60)$ : average growth inhibition $50 \%$ in 60 cancer cell lines (different cancer types), $\Sigma \mathrm{GI}_{50}$ (OvCa): average growth inhibition 50\% in OvCa cell lines.

causes single strand breaks while Topo II with its isoforms $\alpha$ and $\beta$ is responsible for the doublestrand break (Figure 1). ${ }^{5,6}$ Such topoisomerase poisoning leads to cell death by inducing apoptosis. The aza-analogous benzo[c]phenanthridine P8-D6 was synthesized in an optimized four-step process with advantageous physicochemical and cytotoxic properties. ${ }^{7}$ In the National Cancer Institute (NCI)-60 DTP Human Tumor Cell Line screening $49 \mathrm{nM}$ of P8-D6 results in an average growth inhibition of $\left.50 \%\left(\mathrm{GI}_{50}\right)\right)^{7,8}$ The result for OvCa cell lines were $0.12 \mu \mathrm{M}$ compared with cisplatin with $15.25 \mu \mathrm{M}$ or topotecan with $0.23 \mu \mathrm{M}$, respectively.

Cell-based assays are an important pillar in drug development. In addition to traditional twodimensional (2D) monolayer, co-culture and three-dimensional (3D) cell culture have recently gained importance because of greater comparability with in vivo set ups. ${ }^{9}$ Therefore, efficacy studies of P8-D6 were performed on different OvCa cell lines and patient-derived primary cells, compared with single drugs and combinational drug therapy. ${ }^{10-12}$

Preclinical cancer drug development addressed several topics, including target achievement, induction of apoptosis in cancer cells and toxicity in normal cells.

\section{Methods}

\section{Materials}

P8-D6 was synthesized as recently described ${ }^{7}$ and solved in phosphate-buffered saline (PBS). Topotecan, etoposide, cisplatin and doxorubicin were obtained from the UKSH dispensary.

\section{In vitro experiments}

Cell preparation and culture. Human OvCa cell lines A2780, BG-1, Igrov-1, OvCar8, SKOV-3 and fibroblasts Detroit 551 were maintained in RPMI 1640 supplemented with $10 \%$ fetal bovine serum 
(FBS), $\quad 60 \mathrm{IU}(\mu \mathrm{g}) / \mathrm{ml}$ penicillin-streptomycin. SKOV-3luc (firefly luciferase gene) were grown in DMEM (Dulbecco's Modified Eagle Medium), supplemented with $10 \% \mathrm{FBS}, 800 \mu \mathrm{g} / \mathrm{ml}$ neomycin. Primary OvCa cells were isolated from advanced stage $\mathrm{OvCa}$ patients during surgery at first diagnosis (UKSH, Campus Kiel). The tumour cells were extracted from tumour tissue and ascites as described previously. ${ }^{13,14}$ Human ovarian surface epithelial cells (HOSE) (Innoport) were cultivated in OSE medium containing $1 \%$ OEpiCGS, $100 \mathrm{IU}(\mu \mathrm{g}) / \mathrm{ml}$ penicillin-streptomycin. Primary human hepatocytes were isolated from liver tissue under surgery (Uppsala University Hospital), isolated and cultured as described previously. ${ }^{15}$ Cells were grown at $37^{\circ} \mathrm{C}, 5 \% \mathrm{CO}_{2}$ and short tandem repeat profiling ${ }^{16}$ and mycoplasma contamination by MycoAlert ${ }^{\mathrm{TM}}$ (Lonza) were checked.

Informed consent was obtained from all donors, in agreement with the approval from the Institutional Review Board of the UKSH, Campus Kiel (AZ: D578/20) and the Uppsala Regional Ethical Review Board (Ethical Approval 2009/028).

Western blot. Cells were harvested, protein contents determined and SDS-PAGE and western blot analysis was carried out as described previously. ${ }^{17}$ Membranes were incubated with primary antibodies (anti-TopoI 1:500 (Santa Cruz\#sc-271285), anti-TopoII $\alpha / \beta \quad 1: 10000$ (Abcam\#ab109524), anti-HSP 90 1:10000 (Santa Cruz\#sc-13119)) and HRP-labelled antimouse IgG 1:2000 (Santa Cruz\#sc-516102) or HRP-labelled goat anti-rabbit IgG 1:3000 (Elabscience\#E-AB-1003). Chemiluminescence was visualized using ECL Plus Western Blotting Detection System and ChemoStar ECL and Fluorescence Imager (Intas).

Fluorescence imaging. Due to its chemical structure, P8-D6 has fluorescent properties $\left(462_{\mathrm{Ex}} / 530_{\mathrm{Em}}\right)$. In all, 10,000 cells/well were seeded in glass-bottomed 4 -well chamber slides and treated with $10 \mu \mathrm{M}$ P8-D6 or PBS for $30 \mathrm{~min}$, washed with PBS and fixed with acetone $(10 \mathrm{~min}$ at room temperature). Samples were stained by CellTracker ${ }^{\mathrm{TM}}$ Deep Red Dye $\left(5 \mu \mathrm{M}\right.$ at $37^{\circ} \mathrm{C}$ for $15 \mathrm{~min}$ ) and 4',6-diamidin-2-phenylindol (DAPI) $(0.5 \mu \mathrm{g} / \mathrm{ml})$ (Vectashield). Fluorescence imaging was performed using DAPI $\left(350_{\mathrm{EX}} / 470_{\mathrm{Em}}\right)$, FITC $\left(490_{\mathrm{Ex}} / 525_{\mathrm{Em}}\right)$ and Cy5 $\left(649_{\mathrm{Ex}} / 670_{\mathrm{Em}}\right)$ filters. Microscope Axioplan 2 (Carl Zeiss Microscopy), Isis Version 5.8.8. (MetaSystems).
The 2 D viability and apoptosis assay. In all, 10,000 cells/ well were seeded in a 96-well plate (Corning \#3903) and treated for $48 \mathrm{~h}$. The measurement using ApoLive-Glo ${ }^{\text {TM }}$ Multiplex Assay (Promega \#G6410) was performed as described in the instruction (TM325) with a microplate reader (Infinite 200, Tecan). Relative caspase activity calculation: caspase activity divided by the viability (normalized to control). With viability data, dose-response curves were plotted and inhibitory concentration $50 \%\left(\mathrm{IC}_{50}\right)$ values were calculated (GraphPad).

Flow cytometric analysis. Cells were seeded in 6-well plates and treated for $48 \mathrm{~h}$. Cells were harvested and stained as described previously. ${ }^{17}$

The $3 D$ cytotoxicity, viability and apoptosis assay: A2780 (200/well), SKOV-3 (8000/well), OvCar8 (1000/well) and BG-1 (450/well) cells were seeded into a 96-well Ultra-Low Attachment (ULA) plate (Corning \#4520) and grown for $96 \mathrm{~h}$. Then, spheroids were treated for $48 \mathrm{~h}$. Simultaneously, CellTox $^{\mathrm{TM}}$ Green assay (Promega \#G8731) was added and detected $\left(485_{\mathrm{Ex}} 520_{\mathrm{Em}}\right) 24$ and $48 \mathrm{~h}$ after treatment using NYONE $^{\circledR}$ (SYNENTEC). Filters: $\quad \mathrm{BF}_{\mathrm{Ex}} / \mathrm{Green}_{\mathrm{Em}} \quad(530 / 43 \mathrm{~nm}) ; \quad$ Blue $_{\mathrm{Ex}}$ $(475 / 28 \mathrm{~nm}) / \mathrm{Green}_{\mathrm{Em}}(530 / 43 \mathrm{~nm})$. Subsequently, viability and apoptosis were determined by RealTime-Glo ${ }^{\mathrm{TM}} \quad\left(460_{\mathrm{Em}}\right)$ (Promega \#G9711)

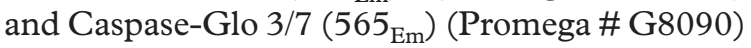
using microplate reader (Infinite 200, Tecan). The measurement was performed according to the instructions. Relative caspase activity: caspase activity divided by the viability (normalized to control). For live-dead staining, cells were grown and treated as described above. Then, $80 \%$ of the medium was removed and replaced with propidium iodide (PI) $(10 \mu \mathrm{g} / \mathrm{ml})$, calcein-AM $(1 \mathrm{mM})$ and hoechst $33342(0.001 \%)$ in medium for $3 \mathrm{~h}$ and imaged by NYONE$^{\circledR}$ (SYNENTEC). Filters: $\mathrm{BF}_{\mathrm{Ex}} / \mathrm{Green}_{\mathrm{Em}}(530 / 43 \mathrm{~nm})$; hoechst 33342: $\mathrm{UV}_{\mathrm{Ex}}$ $(377 / 50 \mathrm{~nm}) /$ Blue $_{\mathrm{Em}}(452 / 45 \mathrm{~nm}) ; \quad$ calcein-AM: Blue $_{\mathrm{Ex}}(475 / 28 \mathrm{~nm}) / \mathrm{Green}_{\mathrm{Em}}(530 / 43 \mathrm{~nm}) ; \mathrm{PI}:$ $\mathrm{Lime}_{\mathrm{Ex}}(562 / 40 \mathrm{~nm}) / \operatorname{Red}_{\mathrm{Em}}(628 / 32 \mathrm{~nm})$.

Scanning electron microscopy (SEM). Spheroids were grown as described above, treated with $1 \mu \mathrm{M}$ P8-D6 and PBS for $48 \mathrm{~h}$, and fixed with $2.5 \%$ glutaraldehyde ( $1 \mathrm{~h}$ room temperature) and then $1 \%$ osmium tetroxide ( $1.5 \mathrm{~h}$ room temperature). Spheroids were dehydrated with ethanol $(25 \%$, $50 \%, 75 \%, 96 \%, 100 \%)$ and air dried using hexamethyldisilazane on charcoal stubs overnight. Then, spheroids were coated with gold and measured with SEM (Phenom XL). 
Co-culture. For co-culture, 40,000 Detroit 551 fibroblasts were seeded into 24 -well plates, and 40,000 A2780 were cultured onto inserts (ThinCert $^{\mathrm{TM}}$ translucent $0.4 \mu \mathrm{m}$ ). Cells were treated for $48 \mathrm{~h}$. Cells were harvested and centrifuged $(10 \mathrm{~min}, 250 \mathrm{~g})$. Cell pellets were resuspended in $25 \mu \mathrm{l}$ medium. Viability and apoptosis were measured using ApoLive-Glo ${ }^{\mathrm{TM}}$ Multiplex Assay.

Hepatotoxicity. Oxidative stress of hepatocytes caused by $48 \mathrm{~h}$ treatment was analysed via dihydroethidium fluorescence. The cell culture protocol was previously described. ${ }^{15}$ Apoptosis was analysed using ApoLive-Glo ${ }^{\text {TM }}$ Multiplex Assay.

Statistical analysis. Statistical tests were performed using GraphPad Prism9 (GraphPad). Gaussian distribution was tested by Shapiro-Wilk normality test. Data of multiple groups were checked with one-way analysis of variance (ANOVA) for statistical significance. Statistically significant differences were assumed at $p$ values $\left.<0.05{ }^{(}\right)$ according to Tukey's multiple comparison and Dunn's method.

\section{Results}

Previous studies showed that P8-D6 functions as dual topoisomerase inhibitor. ${ }^{4,7}$ However, the effectiveness is highly dependent on reaching its nuclear target structure - topoisomerase I/II. In addition to colon cancer cells, ${ }^{4}$ we checked the location of P8-D6 and Topo I and II expression in OvCa. Using fluorescent microscopy, P8-D6 was identified in the nucleus (Additional file $1 \mathrm{~A}$ and B in the Supplemental material). In addition, western blot provided evidence that all cells also express sufficient Topo I and Topo II (Additional file $1 \mathrm{C}$ in the Supplemental material).

\section{P8-D6 is highly effective in OvCa 2D monolayers}

The main aim of this study was to prove efficacy of P8-D6 in OvCa. Therefore, OvCa cell lines (A2780, Igrov-1, BG-1, OvCar8, SKOV-3, SKOV-3luc) were treated with P8-D6, and compared with topotecan, etoposide and cisplatin. Initially, the viability of OvCa cells after $48 \mathrm{~h}$ treatment was measured using an enzymatic assay and $\mathrm{IC}_{50}$-values were determined. P8-D6 exhibits a four times lower $\mathrm{IC}_{50}$-value and is, therefore, significantly more effective than the standard chemotherapeutics (Figure 2(a) and (h); Additional file 2A in the Supplemental material).
A significantly higher increase of apoptosis after $48 \mathrm{~h}$ treatment with P8-D6 compared with its standard therapeutic drugs could be observed in all tested OvCa cell lines by using ApoLive-Glo ${ }^{\mathrm{TM}}$ Multiplex Assay (Figure 2(b), Additional file $2 \mathrm{~B}-\mathrm{F}$ in the Supplemental material) and flow cytometric analyses (Figure 2(d) and (e); Additional file $3 \mathrm{~B}$ in the Supplemental material).

Since P8-D6 is a dual topoisomerase inhibitor, but the reference substances primarily inhibit one of the enzymes only, a combination of a Topo I (topotecan) and a Topo II (etoposide) inhibitor was analysed. Compared with this combination, P8-D6 shows a significantly higher rate of apoptosis (Figure 2(c); Additional file $3 \mathrm{~A}$ in the Supplemental material). To validate the pronounced induction of apoptosis and anti-proliferative effect of P8-D6 in primary cells, we used ex vivo patient-derived cells from tumour tissue and ascites in a translational aspect. A significantly higher rate of anti-proliferative and apoptotic effect was observed in primary cells by P8-D6 compared with comparative substances (Figure 2(f) and (g); Additional file $2 \mathrm{G}$ and $\mathrm{H}$ in the Supplemental material). Altogether, we generated results of a plurality of different cells (established and primary cells) to emphasize the durable and robust effect of P8-D6 (Figure 2(h)).

\section{P8-D6 induce strong effects in 3D target tumour and co-culture model}

The 3D cell cultures are regarded to bridge the gap from 2D to in vivo models, since cell-cell interaction is considerable for the efficacy of a substance. The 3D spheroids mimic the physiological behaviour of solid tumours more closely. ${ }^{10,11}$ Spheroids were generated in ULA plates for $96 \mathrm{~h}$ and subsequently treated with P8-D6 or topotecan. The OvCa spheroids (A2780, SKOV-3, BG-1, OvCar8) showed a decrease in growth behaviour and stability after P8-D6 treatment (Figure 3(a); Additional file $4 \mathrm{~A}-\mathrm{C}$ in the Supplemental material). Cell toxicity was increased in all spheroids by P8-D6 compared with topotecan and PBS (Figure 3(b) and (c); Additional file $4 \mathrm{D}-\mathrm{F}$ in the Supplemental material). Furthermore, P8-D6 exerted a significantly increased pro-apoptotic effect in all spheroids compared with control (Figure 3(d); Additional file $5 \mathrm{~A}-\mathrm{C}$ in the Supplemental material). To visualize the potency of P8-D6 in spheroids, a triple live-dead staining consisting of calcein-AM, PI and hoechst 33342 was used 
(a) Cytotoxicity $\left[\mathrm{IC}_{50}\right]$
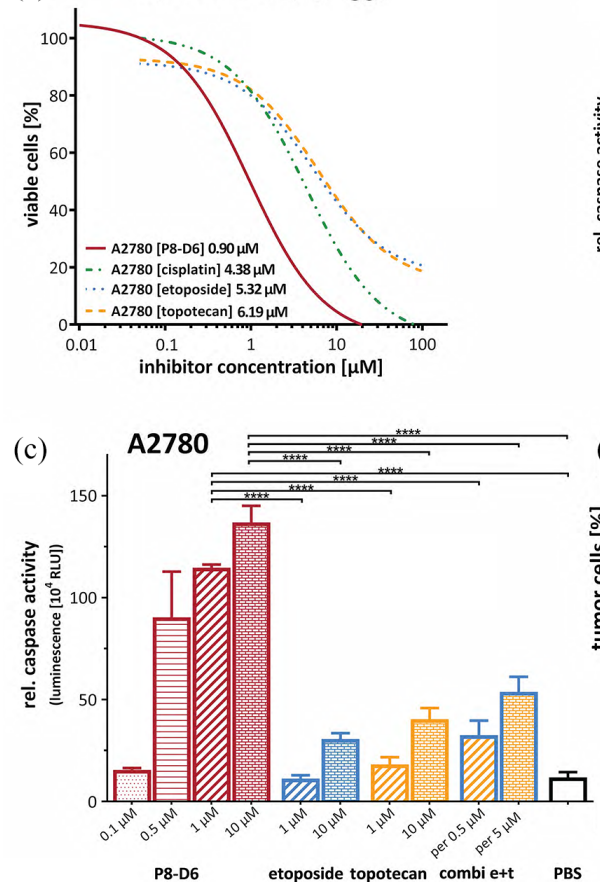

(1)

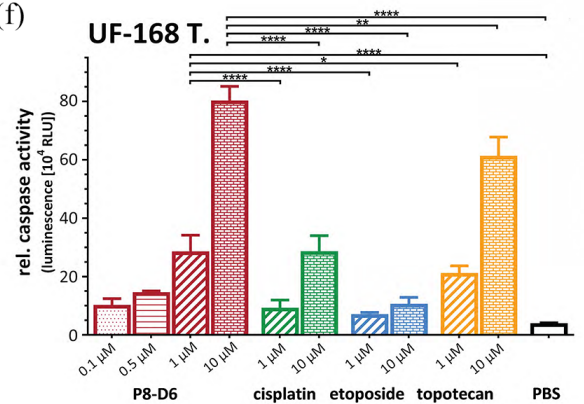

(b) A2780

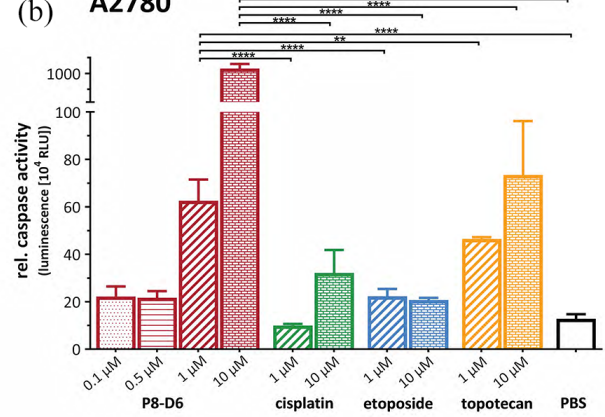

(d) $\mathrm{A2780}$

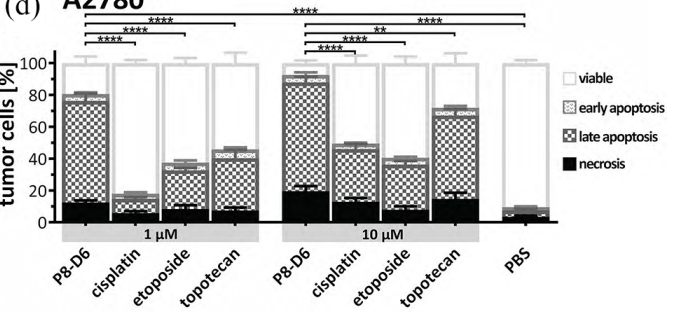

(e) P8-D6 $[1 \mu \mathrm{M}]$ topotecan $[1 \mu \mathrm{M}]$ etoposide $[1 \mu \mathrm{M}]$

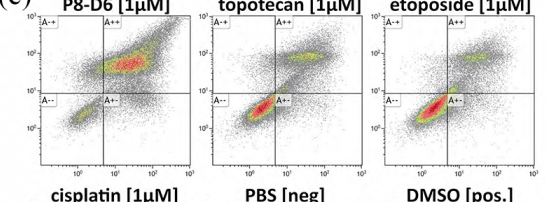

(g)

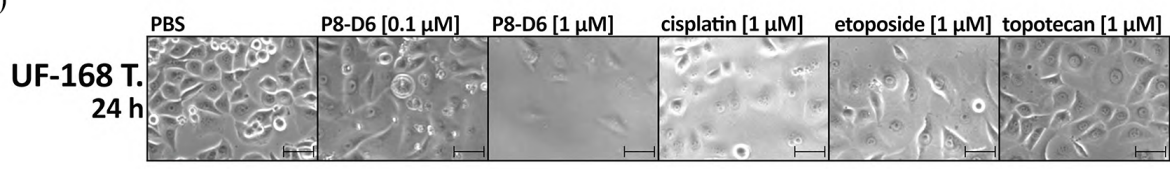

(h)

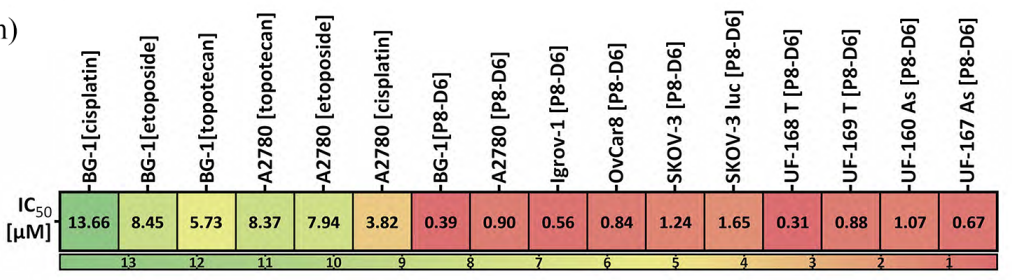

Figure 2. Antitumour responses in OvCa 2D monolayers. A2780 (cell line) and UF-168T (primary cells) were treated with different concentrations of P8-D6, topotecan, etoposide, cisplatin and negative control (PBS) for $48 \mathrm{~h}$. Subsequently, the viability and caspase activity were determined. (a) The $I C_{50}$ values of each cytostatic drug were calculated by using the viability data. (b) The apoptosis is represented as relative caspase activity. (c) To compare the combinatorial apoptotic effect of topotecan (Topo I Inhibitor) and etoposide (Topo II Inhibitor) with P8-D6, a dual topoisomerase inhibitor was performed in A2780. (d) and (e) Flow cytometric analysis of pro-apoptotic effects with Annexin V-PE (An V) and 7AAD staining $(n=6)$. Representative flow cytometry dot plots of treated and stained A2780 cells were done. The mean distribution of viable (An V/7AAD-negative), early apoptotic (An V-positive, 7AAD-negative), late apoptotic/necrotic (An V/7AAD-positive) or necrotic (An V-negative, 7AAD-positive) tumour cells after treatment were calculated (d). (f) For primary OvCa cells (UF-168T) viability and apoptosis were measured. (g) In addition, the anti-proliferative effect after 24-h treatment was evaluated by microscopy. Scale bars, $50 \mu \mathrm{m}$. (h) Heat map of the $\mathrm{IC}_{50}$ values using the viability of all tested OvCa cells. Data are means $+S D$ (standard deviation) one-way ANOVA; ${ }^{*} p<0.05,{ }^{* *} p<0.01,{ }^{* * *} p<0.001,{ }^{* * * *} p<0.0001$. 
(a)

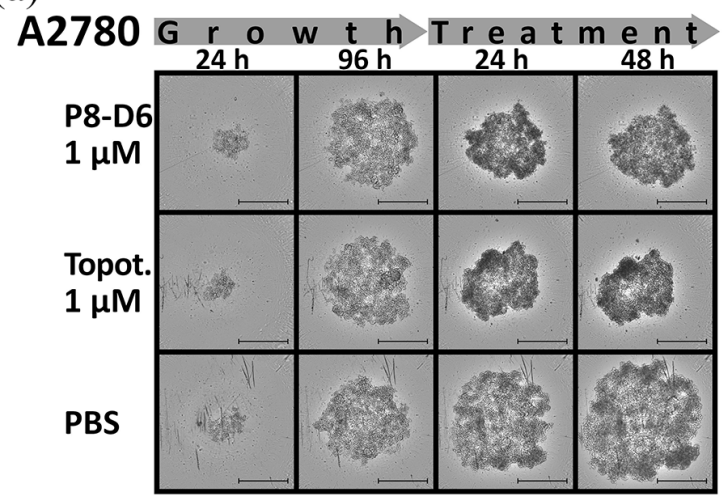

(d)

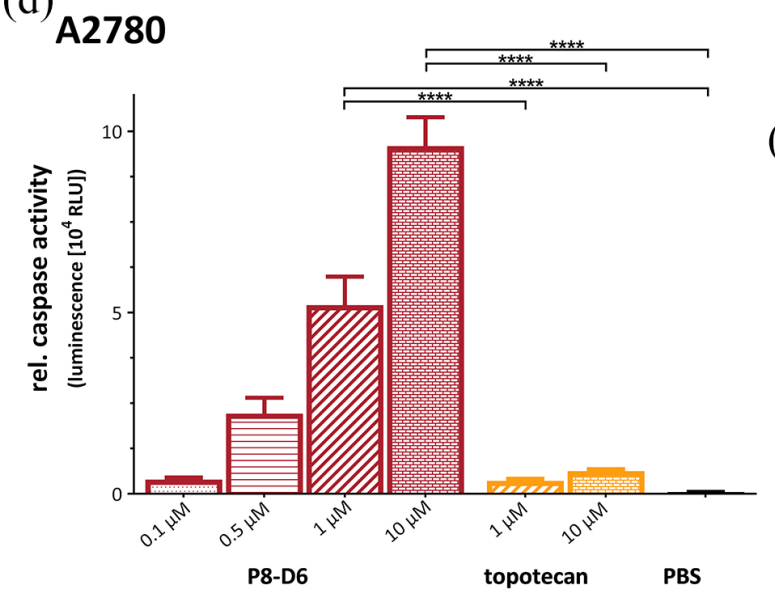

(f)

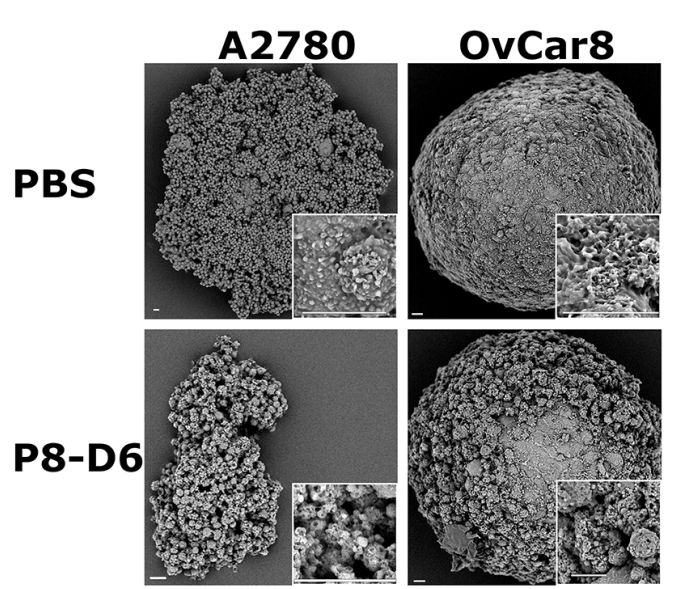

(b)

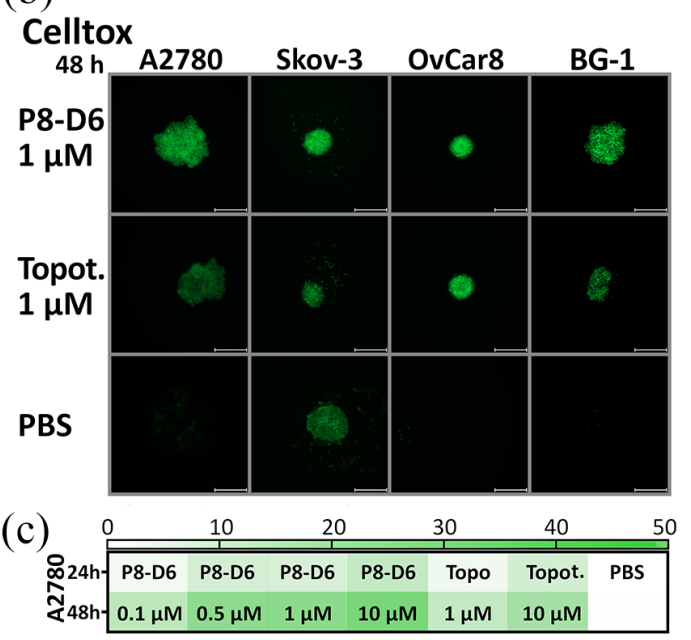

(e)

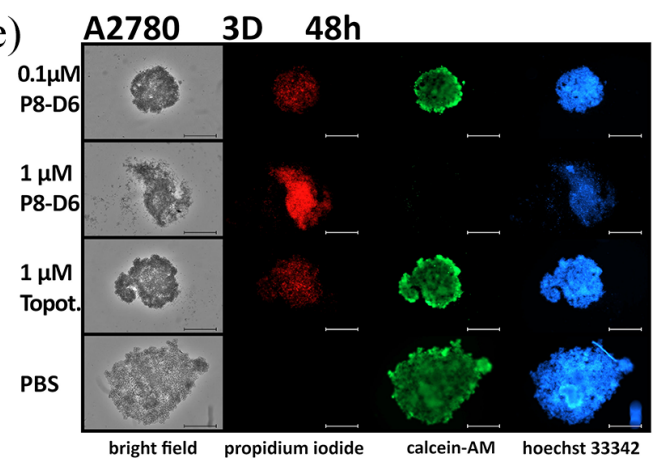

(g) co-culture
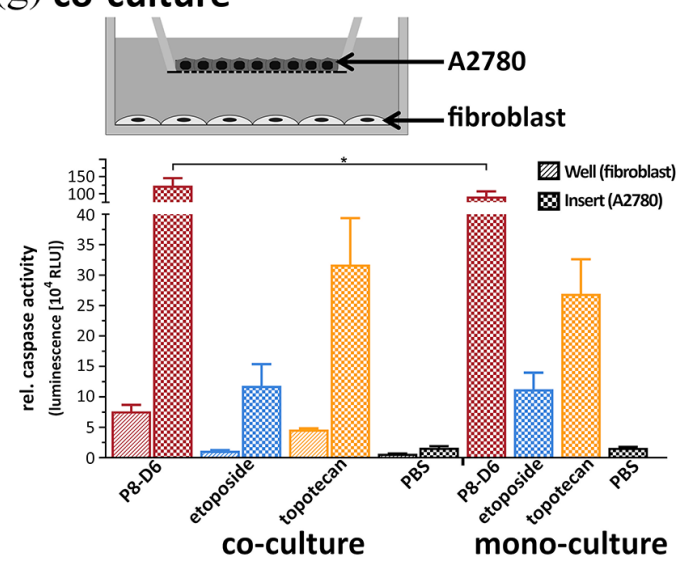

Figure 3. Antitumour properties in 3D spheroids and 2D co-culture. For 3D culture, A2780, SKOV-3, OvCar8 and BG-1 cells were maintained in ULA plates for $96 \mathrm{~h}$ and subsequently treated with P8-D6 $(0.1,0.5,1$ and $10 \mu \mathrm{M})$, topotecan $(1$ and $10 \mu \mathrm{M})$ and PBS for $48 \mathrm{~h}$. (a) Every $24 \mathrm{~h}$ images were generated by microscopy. Scale bars, $500 \mu \mathrm{m}$. (b) and (c) During treatment, the cell toxicity was measured by fluorescence microscope using CellToxTM Green (24 and $48 \mathrm{~h}$ ). Scale bars, $500 \mu \mathrm{m}$. The fluorescence signals for 24 and $48 \mathrm{~h}$ after treatment were quantified (relative fluorescence units RFU) and shown in a heat map (c). (d) After 48-h treatment, the viability and caspase activity were measured in A2780 spheroids. (e) A2780 spheroids were stained after the growth and treatment phase with PI (red), calcein-AM (green) and hoechst 33342 (blue), and measured by microscopy. Scale bars, $500 \mu \mathrm{m}$. (f) SEM images of A2780 and OvCar8 spheroids, which were treated with P8-D6 (1 $\mu \mathrm{M})$ or PBS for $48 \mathrm{~h}$ were taken. Scale bars, $20 \mu \mathrm{m}$. (g) For co-culture experiments, A2780 cells were seeded in 2D monolayers on transwell inserts and fibroblasts on well bottoms. For comparison, mono-cultures were cultured and treated with P8-D6 $(10 \mu \mathrm{M})$, etoposide $(10 \mu \mathrm{M})$, topotecan $(10 \mu \mathrm{M})$ and PBS in the same way. The apoptosis represented as relative caspase activity was measured in A2780 and fibroblasts. Data are means $+S D(n=3)$ one-way ANOVA; ${ }^{*} p<0.05,{ }^{* *} p<0.01,{ }^{* * *} p<0.001,{ }^{* * * *} p<0.0001$. 
(a)

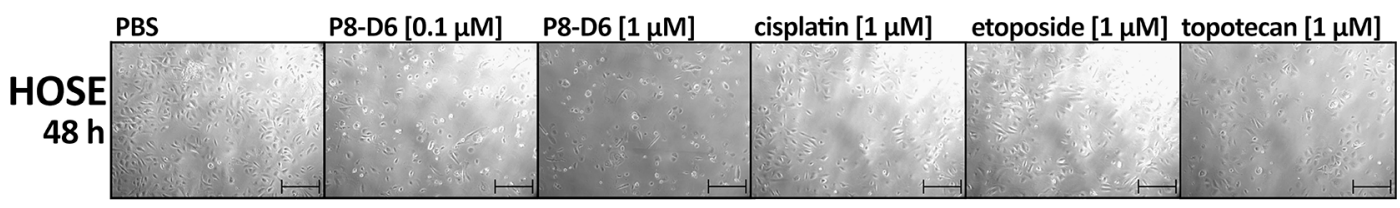

(b)

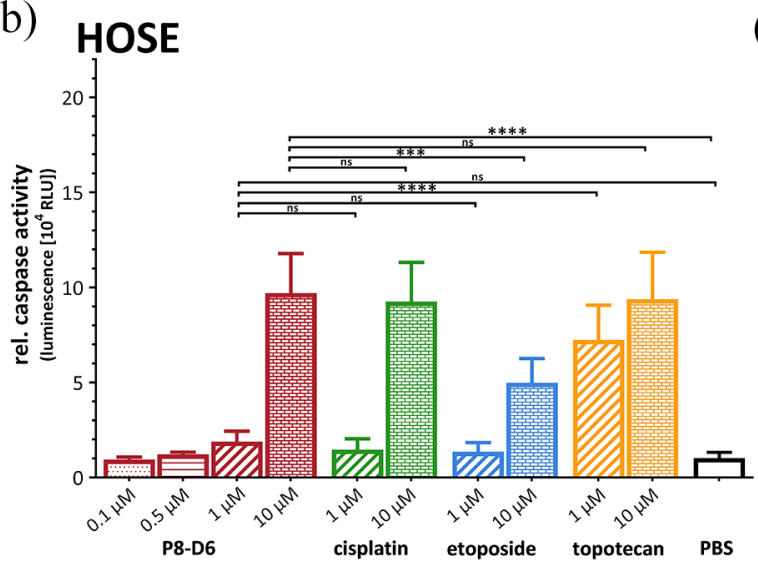

(c) Hepatotoxicity

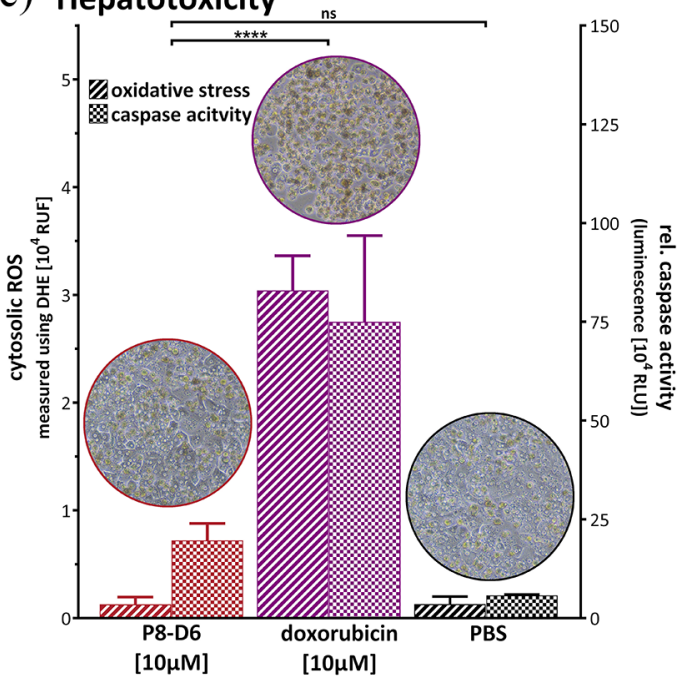

Figure 4. Effects on non-cancer cells. (a) To examine toxic effects of therapy, human ovarian surface epithelial cells (HOSE) were treated with P8-D6, cisplatin, etoposide, topotecan and PBS for $48 \mathrm{~h}$. The anti-proliferative effect after $48 \mathrm{~h}$ treatment was evaluated by microscopy. Scale bars, $100 \mu \mathrm{m}$. (b) The viability and apoptosis were measured after $48 \mathrm{~h}$ treatment in HOSE cells. Data are means $+S D(n=6)$ one-way ANOVA; ${ }^{*} p<0.05$, ${ }^{* *} p<0.01,{ }^{* * *} p<0.001,{ }^{* * * *} p<0.0001$. (c) To investigate the hepatotoxicity, primary human hepatocytes were treated with P8-D6, doxorubicin and PBS for $48 \mathrm{~h}$. Afterwards, microscopy images were taken, and apoptosis and oxidative stress measurements were performed. Data are means $+S D(n=3)$ one-way ANOVA; ${ }^{*} p<0.05$, ${ }^{* *} p<0.01,{ }^{* * *} p<0.001,{ }^{* * * *} p<0.0001$.

(Figure 3(e), Additional file 5D-F in the Supplemental material). The decreased staining of calcein-AM and the increase of cells stained with PI proved the strong cytotoxic effect of P8-D6. In addition, P8-D6 treated cells showed disintegration of the spheroid and a considerably higher number of dead cells than topotecan. A 10-fold lower dose of P8-D6 showed similar results as $1 \mu \mathrm{M}$ topotecan. Moreover, SEM identified surface changes of the spheroids like loss of membrane integrity due to treatment (Figure 3(f), Additional file $4 G$ in the Supplemental material).

In addition, it was investigated whether fibroblast-A2780 co-culture could mediate changes in cancer cell responses to anti-cancer drugs by affecting cell-cell interaction (Figure $3(\mathrm{~g})$ ). Co-culture promotes the apoptosis induction significantly for P8-D6 treated cancer cells compared with mono-culture, while the co-culture treated cancer cells with etoposide and topotecan only exhibited minimal activity differences.

\section{P8-D6 only slightly affects non-cancer cells}

Since side effects often occur as a result of tumour therapy, it is important to determine the toxicity of P8-D6 in preclinical setting on non-cancerous cells such as HOSE. HOSE were treated for $48 \mathrm{~h}$ and the anti-proliferative effect of P8-D6 is only slightly increased compared with the reference (Figure 4(a)). While $1 \mu \mathrm{M}$ P8-D6 is highly effective on cancer cells, no significant increase of apoptosis was measured compared with PBS control in HOSE cells. The application of $10 \mu \mathrm{M}$ P8-D6 shows a similar value as positive controls (Figure 4(b)).

Hepatotoxicity is a common and serious side effect in chemotherapy. By measuring oxidative stress and induction of apoptosis after 48-h 
treatment in primary human hepatocytes, P8-D6 showed no significant difference compared with PBS, while doxorubicin induces significant cell damage effects (Figure 4(c)). P8-D6 proved to be cytotoxic against $\mathrm{OvCa}$ cells without inducing cell death in hepatocytes.

\section{Discussion}

Since OvCa is one of the world's deadliest gynaecological malignancies, there is a high clinical need for the development of new, effective and well-tolerated therapy options with suitable physiochemical properties. ${ }^{1}$ P8-D6 was investigated as a novel dual topoisomerase inhibitor in OvCa cell line and ex vivo patient-derived primary cells in $2 \mathrm{D}, 3 \mathrm{D}$ and co-culture, and was proven to be overall significantly more effective than standard therapeutics and negative control.

Side effects due to chemotherapy have significant impacts on therapy. However, P8-D6 shows only limited toxic effects on normal cells measured by oxidative stress and induces apoptosis. The significantly reduced influences on the normal cells could be explained by the reduced ability of these cells to divide, but these causes have to be investigated furthermore. If efficacy and toxicity studies are related to clinical therapy, P8-D6 induces significantly higher ( $>$ five-fold) apoptosis rates in OvCa than current standards. Thus, it could potentially be used in lower doses than the standard therapeutic agents to achieve the same effect.

Due to the dual topoisomerase inhibition of P8-D6, the question arises whether a combination therapy of two established mono-Topo I/II inhibitors reach the antitumour potency of P8-D6 in OvCa. Previous studies compared the combination of Topo I with Topo II inhibitor versus monotherapy and showed heterogeneous results. ${ }^{18-21}$ This study showed significantly higher induction of apoptosis by P8-D6 when compared with the additive effect of Topo I and Topo II inhibitor combination. So far, the combination of topotecan and etoposide shows no clinical benefit ${ }^{22}$ and is not considered as a clinical standard, whereas the monotherapy of topotecan is proposed to treat in second line therapy. 23,24

A major advantage of a dual topoisomerase inhibitor is the reduced development of resistance through inhibition of both topoisomerases. The inhibition of only one enzyme causes a compensatory upregulation of the other. ${ }^{21,25}$ The effectiveness of dual topo-inhibitors on solid tumours has been shown previously. ${ }^{26,27}$ In clinical trials, however, these drugs showed intolerable side effects (e.g. intoplicine, TA-103, batracylin), such as hepatotoxicity and severe neutropenia. ${ }^{27-33}$ Because of this increased hepatotoxicity with dual topoisomerase inhibitors, ${ }^{34}$ the hepatotoxicity of P8-D6 was determined. Importantly, this study shows that P8-D6 has no relevant effect concerning oxidative stress and apoptosis in human hepatocytes. The mechanism of action is the key benefit and novel about these drugs by affecting both topoisomerases. However, it is a validated target since single Topo inhibitors are already standards in cancer therapy. ${ }^{23,24,35}$

The current therapy for platinum-resistant/refractory OvCa consists mainly of a monotherapy with paclitaxel, gemcitabine or PLD (pegylated liposomal doxorubicin), but cancer control rate is limited. $^{36,37}$ In addition, topotecan is another approved treatment option for platinum-resistant/ refractory OvCa therapy. ${ }^{38,39}$ Hence, some novel drugs are in clinical trials like the tyrosine kinase inhibitor rivoceranib. ${ }^{40}$ The antibody drug conjugate (ADC) Mirvetuximab soravtansine (MIRV) comprises a Folate receptor alpha-binding antibody, cleavable linker and the tubulin-targeting toxin DM4. A Phase III study evaluated the safety and efficacy of MIRV compared with chemotherapy in patients with platinum-resistant OvCa. ${ }^{41}$

A combination of P8-D6 with a tyrosyl-DNA phosphodiesterase inhibitor or a Poly (ADPribose) polymerase (PARP) inhibitor could merit additional consideration. These enzymes are involved in the repair mechanisms of the TopoDNA complex and would possibly have a further positive effect in apoptosis induction. ${ }^{42,43}$ Liposomal formulations could also be possible further approaches for P8-D6. This special drug formulation would significantly increase the selectivity of the active ingredient and thus contribute to better tolerability of the active ingredient, since topoisomerase are ubiquitous in cells and can thus trigger slight side effects there, as known with other cytostatics.

\section{Conclusion}

In summary, P8-D6 has promising antitumour properties in 2D, 3D and co-culture in OvCa. It has fewer effects on normal ovarian cells and hepatocytes than its references. To sum up, P8-D6 is a strong and rapid inductor of apoptosis 
and warrants further development. Further in vivo experiments for P8-D6 are needed to verify antitumour effects also for complex multiorgan systems. In addition, further studies on other side effects that could lead to dose-limitation should be performed.

\section{Acknowledgements}

The authors thank Dr Marie Hellfritzsch for assistance with SEM imaging, and Sigrid Hamann and Catharina Verkooyen for technical assistance.

\section{Author contributions}

Conceptualization: IF and DOB; investigation: IF; methodology: IF, NH, MÖ; resources: TNS and $\mathrm{BC}$; supervision: $\mathrm{IF}, \mathrm{BC}$ and $\mathrm{DB}$; writing original draft preparation: IF; writing - review and editing: TNS, NH, MÖ, PA, BC and DOB. All authors have read and agreed to the published version of the manuscript.

\section{Conflict of interest statement}

The authors declared no potential conflicts of interest with respect to the research, authorship and/or publication of this article.

\section{Funding}

The authors received no financial support for the research, authorship and/or publication of this article.

\section{Ethics approval and consent to participate}

Institutional Review Board Statement: The study was conducted according to the guidelines of the Declaration of Helsinki, and approved by the Institutional Review Board of the University Medical Centre Schleswig-Holstein, Campus Kiel (AZ:D578/20) and the Uppsala Regional Ethical Review Board (Ethical Approval no. 2009/028). Informed consent was obtained from all subjects involved in the study.

\section{Supplemental material}

Supplemental material for this article is available online.

\section{References}

1. Siegel RL, Miller KD and Jemal A. Cancer statistics, 2020. CA Cancer f Clin 2020; 70: 7-30.

2. Ledermann JA. First-line treatment of ovarian cancer: questions and controversies to address. Ther Adv Med Oncol 2018; 10: 1-8.
3. Chandra A, Pius C, Nabeel M, et al. Ovarian cancer: current status and strategies for improving therapeutic outcomes. Cancer Med 2019; 8: 7018-7031.

4. Aichinger G, Lichtenberger F-B, Steinhauer TN, et al. The aza-analogous benzo[c]phenanthridine P8-D6 Acts as a dual topoisomerase I and II poison, thus exhibiting potent genotoxic properties. Molecules 2020; 25: 1524.

5. Pommier Y, Barcelo JM, Rao VA, et al. Repair of topoisomerase I-mediated DNA damage. Prog Nucleic Acid Res Mol Biol 2006; 81: 179-229.

6. Deweese JE and Osheroff N. The DNA cleavage reaction of topoisomerase II: Wolf in sheep's clothing. Nucleic Acids Res 2009; 37: 738-748.

7. Meier C, Steinhauer TN, Koczian F, et al. A dual topoisomerase inhibitor of intense pro-apoptotic and antileukemic nature for cancer treatment. Chem Med Chem 2017; 12: 347-352.

8. National Cancer Institute (NCI). DTP homepage: Cancer Screen 09/2014: NCI-60 DTP Human Tumor Cell Line Screen [The National Cancer Institute (NCI) is gratefully acknowledged for its excellent screening service.], 2014, http://dtp.nci.nih.gov

9. Edmondson R, Broglie JJ, Adcock AF, et al. Three-dimensional cell culture systems and their applications in drug discovery and cell-based biosensors. Assay Drug Dev Technol 2014; 12: 207-218.

10. Goodman TT, Olive PL and Pun SH. Increased nanoparticle penetration in collagenase-treated multicellular spheroids. Int $\mathcal{F}$ Nanomedicine 2007; 2: 265-274.

11. Kimlin LC, Casagrande $G$ and Virador VM. In vitro three-dimensional (3D) models in cancer research: an update. Mol Carcinog 2013; 52: 167-182.

12. Lagies S, Schlimpert M, Neumann S, et al. Cells grown in three-dimensional spheroids mirror in vivo metabolic response of epithelial cells. Commun Biol 2020; 3: 1-10.

13. Kurbacher CM, Korn C, Dexel S, et al. Isolation and culture of ovarian cancer cells and cell lines. Methods Mol Biol 2011; 731: 161-180.

14. Hedemann N, Herz A, Schiepanski JH, et al. ADAM17 inhibition increases the impact of cisplatin treatment in ovarian cancer spheroids. Cancers 2021; 13: 2039.

15. Ölander M, Wiśniewski JR, Flörkemeier I, et al. A simple approach for restoration of differentiation and function in cryopreserved human hepatocytes. Arch Toxikol 2018; 93: 819-829. 
16. Huang X, Weimer J, von Wurmb-Schwark $\mathrm{N}$, et al. Alteration of STR profiles in ovarian carcinoma cells during primary culture. Arch Gynecol Obstet 2016; 294: 369-376.

17. Plitzko B, Havemeyer A, Kunze T, et al. The pivotal role of the mitochondrial amidoxime reducing component 2 in protecting human cells against apoptotic effects of the base analog N6-hydroxylaminopurine. F Biol Chem 2015; 290 : 10126-10135.

18. Kaufmann SH. Antagonism between camptothecin and topoisomerase II-directed chemotherapeutic agents in a human leukemia cell line. Cancer Res 1991; 51: 1129-1136.

19. Bertrand RO', O'Connor PM, Kerrigan D, et al. Sequential administration of camptothecin and etoposide circumvents the antagonistic cytotoxicity of simultaneous drug administration in slowly growing human colon carcinoma HT-29 cells. Eur f Cancer 1992; 28A: 743-748.

20. Camacho KM, Kumar S, Menegatti S, et al. Synergistic antitumor activity of camptothecindoxorubicin combinations and their conjugates with hyaluronic acid. F Control Release 2015; 210: 198-207.

21. van Gijn R, Lendfers RRH, Schellens JHM, et al. Dual topoisomerase I/II inhibitors. F Oncol Pharm Pract 2000; 6: 92-108.

22. Sehouli J, Sommer H, Klare P, et al. A randomized multicenter phase III trial of topotecan monotherapy versus topotecan + etoposide versus topotecan + gemcitabine for second-line treatment of recurrent ovarian cancer. $\mathcal{F}$ Clin Oncol 2006; 24: 5030.

23. Abudou M, Zhong D, Wu T, et al. Topotecan for ovarian cancer. Cochrane Database Syst Rev 2008; 2008: CD005589.

24. Markman M. Topotecan as second-line therapy for ovarian cancer: dosage versus toxicity. Oncologist 2005; 10: 695-697.

25. Aronson JK (eds). Side effects of drugs annual 27: Cytostatic drugs. 1st ed. Amsterdam: Elsevier Science, 2004.

26. Skok Zidar Ž N, Kikelj D and Ilaš J. Dual inhibitors of human DNA topoisomerase II and other cancer-related targets. F Med Chem 2020; 63: 884-904.

27. Denny WA and Baguley BC. Dual topoisomerase I/II inhibitors in cancer therapy. Curr Top Med Chem 2003; 3: 339-353.

28. van Gijn R, ten Bokkel Huinink WW, Rodenhuis $\mathrm{S}$, et al. Topoisomerase I/II inhibitor intoplicine administered as a $24 \mathrm{~h}$ infusion: phase I and pharmacologic study. Anticancer Drugs 1999; 10: 17-23.

29. Abigerges D, Armand JP, Chabot GG, et al. Phase I and pharmacology study of intoplicine (RP60475; NSC 645008), novel topoisomerase I and II inhibitor, in cancer patients. Anticancer Drugs 1996; 7: 166-174.

30. Ewesuedo RB, Iyer L, Das S, et al. Phase I clinical and pharmacogenetic study of weekly TAS-103 in patients with advanced cancer. $\mathcal{F}$ Clin Oncol 2001; 19: 2084-2090.

31. Salerno S, Da Settimo F, Taliani S, et al. Recent advances in the development of dual topoisomerase I and II inhibitors as anticancer drugs. Curr Med Chem 2010; 17: 4270-4290.

32. Kummar S, Gutierrez ME, Anderson LW, et al. Pharmacogenetically driven patient selection for a first-in-human phase I trial of batracylin in patients with advanced solid tumors and lymphomas. Cancer Chemother Pharmacol 2013; 72: 917-923.

33. Mucci-LoRusso P, Polin L, Bissery MC, et al. Activity of batracylin (NSC-320846) against solid tumors of mice. Invest New Drugs 1989; 7: 295-306.

34. King PD and Perry MC. Hepatotoxicity of chemotherapy. Oncologist 2001; 6: 162-176.

35. Bookman MA, Brady MF, McGuire WP, et al. Evaluation of new platinum-based treatment regimens in advanced-stage ovarian cancer: a phase III trial of the gynecologic cancer intergroup. F Clin Oncol 2009; 27: 1419-1425.

36. Ding D and Kong W-m. Analysis of relative factors of bone marrow suppression after chemotherapy with carboplatin and paclitaxel on the patients with ovarian cancer. Zhonghua Fu Chan Ke Za Zhi 2011; 46: 188-192.

37. Li X-R, Zhu Y, Zhang G-N, et al. The impact of pegylated liposomal doxorubicin in recurrent ovarian cancer: an updated meta-analysis of randomized clinical trials. $\mathcal{F}$ Ovar Res 2021; 14: 9 .

38. Rowinsky EK, Grochow LB, Hendricks CB, et al. Phase I and pharmacologic study of topotecan: a novel topoisomerase I inhibitor. $\mathcal{F}$ Clin Oncol 1992; 10: 647-656.

39. Abushahin F, Singh DK, Lurain JR, et al. Weekly topotecan for recurrent platinum resistant ovarian cancer. Gynecol Oncol 2008; 108: 53-57. 
40. Miao M, Deng G, Luo S, et al. A phase II study of apatinib in patients with recurrent epithelial ovarian cancer. Gynecol Oncol 2018; 148: 286-290.

41. Moore K, Oza A, Colombo N, et al. FORWARD I (GOG 3011): a phase III study of mirvetuximab soravtansine, a folate receptor alpha (FRa)targeting antibody-drug conjugate (ADC), versus chemotherapy in patients (pts) with platinum- resistant ovarian cancer (PROC). Ann Oncol 2019; 5: 403.

42. Murai J and Pommier Y. PARP trapping beyond homologous recombination and platinum sensitivity in cancers. Annu Rev Cancer Biol 2019; 3: 131-150.

43. Hall SR and Goralski KB. ZATT, TDP2, and SUMO2: breaking the tie that binds TOP2 to DNA. Transl Cancer Res 2018; 7: 439-444.
Visit SAGE journals online journals.sagepub.com/ home/tam

@AGE journals 